\title{
论文
}

\section{短程高效回收废锂离子电池中铜金属的技术及 机理研究}

\author{
祖丽德孜, 李金惠，曾现来* \\ 清华大学环境学院, 固体废物处理与环境安全教育部重点实验室, 北京 100084 \\ *E-mail: xlzeng@tsinghua.edu.cn \\ 收稿日期: 2017-11-08; 接受日期: 2018-06-14; 网络版发表日期: 2018-08-02 \\ 中国博士后科学基金特别资助项目(编号: 2016T90104)、清华大学大学生研究训练计划项目和清华大学亚洲研究中心资助
}

\begin{abstract}
摘要信息通讯技术和电动汽车产业的快速发展产生了大量的废锂离子电池, 其资源化回收成为资源可持续利 用及环境保护的重要手段。但传统的回收过程工艺繁琐, 资源化效率较低阻碍了产业的良性发展. 为了缩短废锂 离子电池的资源化工艺, 选择离子液体作为加热介质, 通过熔化黏结剂, 分离铜箔和负极材料实现对废锂离子电 池中铜金属的回收. 回收过程发生急剧的热对流和热传导过程, 利用搅拌机快速运转强化了热对流的过程和机械 力分离正极材料, 基于传热理论傅里叶定律建立了热传递过程数学模型. 通过详细实验研究发现, 当加热温度 $180^{\circ} \mathrm{C}$ 、搅拌速度 $350 \mathrm{r} / \mathrm{min}$ 、停留时间 $30 \mathrm{~min}$, 可以实现铜金属的短程高效回收. 本技术的研发大大促进了铜铝 金属的高效回收, 打通了废锂离子电池的闭环供应链.
\end{abstract}

关键词废锂离子电池, 资源化, 铜金属, 离子液体, 傅里叶定律

\section{1 引言}

随着信息通讯技术的快速发展和电动汽车产业的 兴起, 逐步产生了大量的产品类废物, 其中废理离子电 池已成为产生量增长最快的废物流之一 ${ }^{[1,2]}$. 据估计, 我国废锂离子电池产生量2015年为10万吨，但2020年 将超过 50 万吨 ${ }^{[3]}$. 尽管废锂离子电池当前未列入危险 废物管理的目录, 但由于废锂离子电池经常残存部分 电量和含有具有毒性的电解质溶液, 粗放的处理可能 引起爆炸、火灾, 存在严重的环境风险 ${ }^{[4]}$. 而且, 废锂 离子电池含有丰富的金属资源，大约含有 $10 \%$ 金属
铜、 $10 \%$ 金属铝、 $8 \%$ 钴和 $2 \%$ 锂 $^{[5]}$. 因而，对废锂离子 电池进行资源化、无害化研究存在重要的理论及现实 意义.

锂离子电池由外壳和内部电芯构成, 外壳一般是 不锈钢、铝、镀镍金属钢壳或塑料外壳. 电池内部的 阳极是由铜箔和碳粉通过黏结剂混合制成，而有机黏 结剂一般为聚合物聚偏四氟乙烯 $(\mathrm{PVDF})^{[6]}$. 物理结构 的致密性和紧凑型制约了回收处理，而显著的分层结 构又为其拆解分离资源化提供可能.

过去已开展了大量研究废锂离子电池的资源化, Chen等人 ${ }^{[7]}$ 开发了高温 $\left(650^{\circ} \mathrm{C}\right)$ 处理工艺，修复正极材

引用格式: 祖丽德孜, 李金惠, 曾现来. 短程高效回收废锂离子电池中铜金属的技术及机理研究. 中国科学: 技术科学, 2018, 48: 991-998 Juldez N, Li J H, Zeng X L. Short-cut and high-efficiency process to recycle copper from spent lithium-ion battery (in Chinese). Sci Sin Tech, 2018, 48: 991-998, doi: 10.1360/N092017-00385 
料满足再利用要求，Wang等人 ${ }^{[8]}$ 开发了基于机械化学 途径正极材料回收的友好工艺, Zhang等人 ${ }^{[9]}$ 以生产过 程中边角料为原料开发了高温加热 $\left(800^{\circ} \mathrm{C}\right)$ 处理再生 工艺, $\mathrm{GaO}^{\text {等人 }}{ }^{[10]}$ 开发以碳酸锂回收的湿法冶金闭路 循环工艺，Xiao等人 ${ }^{[11]}$ 开发了真空冶金回收碳酸锂的 工艺. 针对铜金属的回收，多数是通过破碎、分选以 及湿法冶金或火法冶金获得，该过程工艺繁琐，耗能 很高, 且回收率多受工艺条件限制 ${ }^{[12,13]}$. 如何快速剥离 废锂离子电池阳极，高效分离铜箔与负极材料仍然是 亟待解决的关键技术问题. 离子液体具有难挥发、液 程范围宽、不可燃等优点，在许多领域均得到广泛应 用 ${ }^{[14]}$. 离子液体也在废锂离子电池领域得到应用，并 成功分离了正极材料和铝箔，回收了钴酸锂材料和金 属铝 ${ }^{[15]}$. 本文将基于这些研究，猜想将拆解的阳极置 于加热的离子液体中, 通过熔化PVDF黏结剂, 分离铜 箔和负极材料，并在此基础上探究资源化过程的最佳 工艺条件.

\section{2 实验}

\section{1 供试材料的基本特征}

废锂离子电池主要产生于消费电子和电动汽车, 二者结构相似，本研究选择来自手机的废锂离子电池 作为研究对象, 尺寸大小约为 $200 \mathrm{~mm} \times 160 \mathrm{~mm}$. 为了 防止短路和自燃，所有样品都首先通过盐水充分放电， 然后手工拆解为阴极、阳极、外壳、有机隔膜等. 每 次取阳极部分20 30 g进行实验, 平行实验 2 次. 分析测 试其物理组成见图1，阳极部分(包括负极材料和铜箔) 占 $47 \%$ ，阴极部分(包括正极材料和铝簿)占 $29 \%$ ，外壳 部分占 $21 \%$, 塑料部分占 $3 \%$.

对于环绕铜䈃的负极材料碳粉, 热传导系数 $\lambda$ 、密 度 $\rho$ 、比热容 $c_{\mathrm{p}}$ 和尺寸等主要特征如表 1 所示 ${ }^{[15]}$.

本文选用的离子液体为1-丁基-3-甲基咪唑四氟硼
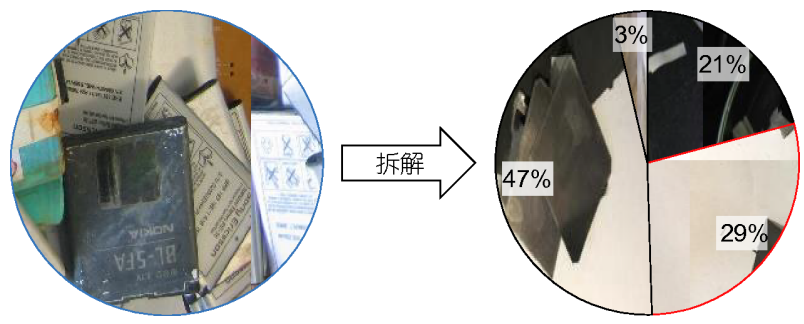

图 1 (网络版彩图)废锂离子电池的物理组成(w.t.)
表 1 负极材料碳粉的物理性质

\begin{tabular}{cc}
\hline 特征 & 数值 \\
\hline 负极材料的厚度 $(\mu \mathrm{m})$ & 80 \\
热传导系数 $\lambda\left(\mathrm{w} /\left(\mathrm{m}{ }^{\circ} \mathrm{C}\right)\right)$ & 129 \\
密度 $\rho\left(\mathrm{g} / \mathrm{cm}^{3}\right)$ & 2.16 \\
比热容 $c_{\mathrm{p}}\left(\mathrm{J} /\left(\mathrm{kg}{ }^{\circ} \mathrm{C}\right)\right)$ & 710 \\
表面热传递系数 $h\left(\mathrm{w} /\left(\mathrm{m}{ }^{\circ} \mathrm{C}\right)\right)$ & 0.02 \\
\hline
\end{tabular}

酸盐([BMIm] $\left.\mathrm{BF}_{4}\right)$, 初始纯度 $>99 \%$, 具有较宽的液程温 度范围、适宜的黏度、良好的水溶性、易合成、价格 便宜等优点 ${ }^{[16]}$, 其基本特征见表 2 所示.

\section{2 实验装置及操作步骤}

实验过程在油浴锅内完成，油浴锅由主体、热电 偶、加热丝和搅拌器组成, 其中搅拌器为外置动力摚 拌器(JJ-1A 精密定时电动搅拌器), 相比磁力搅拌可提 供更大机械力.

实验操作及分析步骤包括：准备实验材料、制作 阳极样品, 并称重; 将离子液体置于油浴锅, 调节温 度、搅拌器转速等参数; 启动油浴加热, 加热到设定 温度; 将清洁处理后的实验材料置于油浴锅，进行加 热分离; 实验完成后关闭油浴锅，回收实验样品和产 物. 实验完成后关闭油浴锅, 冷却后将产生的铜箔取 出, 利用蒸馏水清洗, 清洗液可再次使用; 清洗的铜箔 烘干至恒重, 记录处理后铜箔特征, 称重; 离子液体中 的负极材料通过沉淀分离, 清洗、烘干后获得.

\section{3 正交实验设计}

根据Zeng和 $\mathrm{Li}^{[15]}$ 研究, 可知在加热离子液体拆解 废锂离子电池的过程中, 影响金属剥离的潜在因素主 要包括离子液体的加热温度、阳极部分在离子液体中 的停留时间以及转子转速等. 另外, PVDF黏结剂的分 子链间排列紧密, 密度为 $1.17 \sim 1.79 \mathrm{~g} / \mathrm{cm}^{3}$, 热变形温度

表 2 选用离子液体的基本特征

\begin{tabular}{cc}
\hline 参数 & 数值 \\
\hline 化学结构 & $-81 \sim 400$ \\
液程温度范围 $\left({ }^{\circ} \mathrm{C}\right)$ & 1.2 \\
密度 $\rho\left(\mathrm{g} / \mathrm{cm}^{3}\right)$ & 78.5 \\
黏度系数 $(\mathrm{mPa} \mathrm{s})$ & 0.173 \\
电导率 $(\mathrm{S} / \mathrm{m})$ & \\
\hline
\end{tabular}


$112 \sim 145^{\circ} \mathrm{C}$, 熔化温度为 $172^{\circ} \mathrm{C}^{[17]}$.

为了获取剥离处理回收铜金属的最优条件，基于 三因素三水平进行正交实验设计如表3所示.

\section{3 铜箔与负极材料剥离过程理论计算}

\section{1 剥离过程及其热传递机理}

当将阳极样品材料置入加热的离子液体中时, 热 量将在其表面和内部传递. 离子液体强搅拌条件下, 阳极上表面和下表面水平方向的热对流剧烈, 致使样 品的外部温度快速稳定和一致; 阳极垂直方向发生着 热传导, 带动了负极材料内部和PVDF的升温. 而且, 随着摚拌, 样品材料随着液体一起运动, 加速了传热 过程, 促进了铜箔与负极材料的剥离. 图 2 说明了废理 离子电池阳极置于加热的离子液体中的热传递过程, 阳极主要发生了热传导和热对流, 其中热对流主要发 生于阳极的周围, 热传导主要发生于负极材料、 PVDF和铜箔内. 当加热温度达到 $172^{\circ} \mathrm{C}$ 时, PVDF开始 熔化, 负极材料和铜箔逐渐分离, 一段时间后直至二者 全部分开, 负极材料与铜馢剥离, PVDF进入溶液.

\section{2 剥离过程的时间}

根据传热过程分析，负极材料和铜䇴的剥离时间 可以通过 3 个过程确定：传热达到热平衡、黏结剂熔 化、负极材料与铜箔剥离等，其中负极材料内部热传 递直至温度平衡时间可通过傅里叶定律表达，因而离 子液体加热拆解废锂离子电池阳极的时间可通过式 (1)和(2)确定.

$t=t_{1}+t_{2}+t_{3}$,
表 3 正交实验设计

\begin{tabular}{cccc}
\hline 实验序号 & 加热温度 $\left({ }^{\circ} \mathrm{C}\right)$ & 停留时间 $(\mathrm{min})$ & $\begin{array}{c}\text { 搅拌速度 } \\
(\mathrm{r} / \mathrm{min})\end{array}$ \\
\hline 1 & 170 & 20 & 250 \\
2 & 170 & 25 & 300 \\
3 & 170 & 30 & 350 \\
4 & 175 & 20 & 300 \\
5 & 175 & 25 & 350 \\
6 & 175 & 30 & 250 \\
7 & 180 & 20 & 350 \\
8 & 180 & 25 & 250 \\
9 & 180 & 30 & 300 \\
\hline
\end{tabular}

$\frac{\partial T}{\partial t_{1}}=\frac{\lambda}{\rho c_{\mathrm{p}}}\left[\frac{\partial^{2} T}{\partial x^{2}}+\frac{\partial^{2} T}{\partial y^{2}}+\frac{\partial^{2} T}{\partial z^{2}}\right]+\frac{q_{\mathrm{c}}}{\rho c_{\mathrm{p}}}$,

式中, $t$ 为剥离需要的时间 $(\mathrm{s}), t_{1}$ 为温度平衡需要的时间 $(\mathrm{s}), t_{2}$ 为平衡后的PVDF黏结剂熔化时间 ( $\left.\mathrm{s}\right), t_{3}$ 为负极材 料的脱落时间 $(\mathrm{s}), T$ 为离子液体的加热温度 $\left({ }^{\circ} \mathrm{C}\right), \lambda$ 为碳 粉热传导系数 $\left(\mathrm{W} /\left(\mathrm{m}{ }^{\circ} \mathrm{C}\right)\right), x, y, z$ 为直角坐标系三维方 向, $q_{\mathrm{c}}$ 为过程反应热速率 $(\mathrm{J}), \rho$ 为碳粉的密度 $\left(\mathrm{kg} / \mathrm{m}^{3}\right), c_{\mathrm{p}}$ 为恒压条件下的碳粉比热容 $\left(\mathrm{J} /\left(\mathrm{kg}{ }^{\circ} \mathrm{C}\right)\right)$.

\section{3 铜䇴和负极材料剥离过程的机理特征}

利用加热离子液体对负极材料和铜箔的剥离过程 中, PVDF层只有 $80 \mu \mathrm{m}$, 当加热温度达到PVDF熔点时 将快速熔化, 强外力搅拌下致负极材料迅速脱落, 因而 $t_{2} \approx 0, t_{3} \approx 0$, 即 $t \approx t_{1}$; 热传导主要发生在PVDF层上部(经 负极材料)和下部(经负极材料和铜䈃)的 $x$ 方向, $y, z$ 方 向可以忽略不计，实际上阳极材料内部呈上下对称分

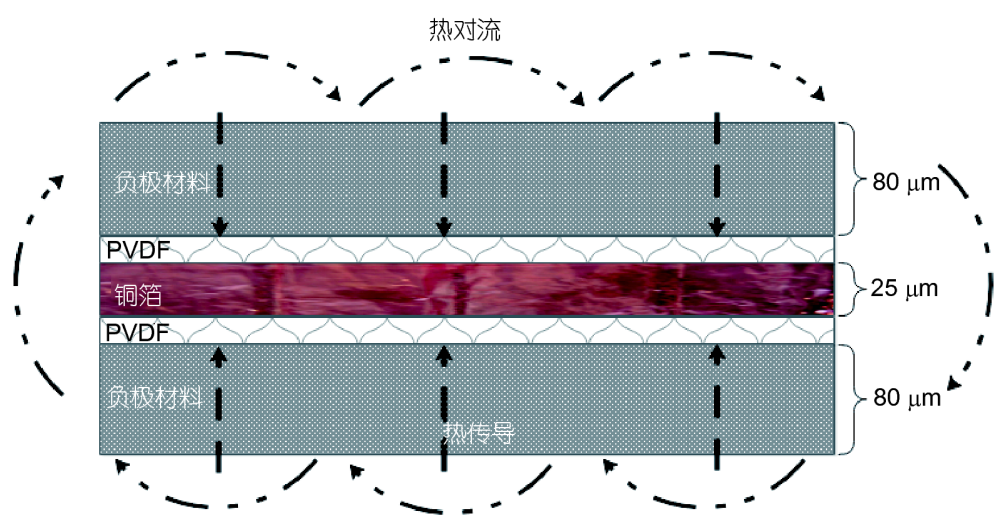

图 2 (网络版彩图)废锂离子电池阳极在热介质处理中的传热过程 
布(图2)，只考虑经上部的热传导计算; 由于负极材 料、铜䇴和离子液体之间没有显著的化学反应，不会 产生热效应, 因而 $q_{\mathrm{c}}=0$. 则式(2)可简化为

$\frac{\partial T}{\partial t_{1}}=\frac{\lambda}{\rho c_{\mathrm{p}}} \times \frac{\partial^{2} T}{\partial x^{2}}$.

将表1中的参数代入, 可得到

$\frac{\partial T}{\partial t_{1}}=8.411 \times 10^{-2} \times \frac{\partial^{2} T}{\partial x^{2}}$.

同时考虑边界约束条件为

$$
\begin{cases}T_{0}=25, & \left(t_{1}=0\right), \\ \frac{\partial T}{\partial x}=0, & (x=0), \\ -129 \times \frac{\partial T}{\partial x}=0.02 \times\left(25-T_{\infty}\right), & (x=80),\end{cases}
$$

式中, $T_{0}$ 为离子液体的初始温度 $\left({ }^{\circ} \mathrm{C}\right), T_{\infty}$ 为离子液体设 定的加热温度 $\left({ }^{\circ} \mathrm{C}\right)$.

基于偏微分方程分离变量的方法和上述边界条
件, 求解式(4), 则温度的分布特征数学函数为

$T\left(x, t_{1}\right)=T_{\infty}+\cos (6.27 x) \mathrm{e}^{-3.31 t_{1}}\left(25-T_{\infty}\right)$.

当离子液体加热的到 $175^{\circ} \mathrm{C} \sim 185^{\circ} \mathrm{C}$ 时，选择 $T_{\infty}=175^{\circ} \mathrm{C}, T_{\infty}=180^{\circ} \mathrm{C}, T_{\infty}=185^{\circ} \mathrm{C}$, 利用数学软件Mathematica 10.0 计算，可得负极材料内部的温度分布如 图3(a) (c)，快速的增长趋势说明负极材料变薄、加热 时间的延长将加剧了阳极内部温度的上升. 相比之下, 由于材料厚度很小 (最大只有 $80 \mu \mathrm{m}$ ), 因而负极材料层 的厚度对阳极内部温度上升的影响不明显. 加热大概 $120 \mathrm{~s}$ 后，热量传递到阳极中心的铜箔，使其温度达到 与离子液体平衡. 加热时间和加热温度关系可通过式 (6)表示, 变化曲线如图3(d)所示. 当设定的加热温度在 $172^{\circ} \mathrm{C}$ 以上时，平衡时间随加热温度快速下降，当加热 温度为 $175^{\circ} \mathrm{C}$ 时, 平衡时间为 $1.2 \mathrm{~min}$, 但当加热温度为 $185^{\circ} \mathrm{C}$ 时, 平衡时间变为不超过 $0.8 \mathrm{~min}$.

$t_{1}=-0.302 \times \ln \frac{172-T_{\infty}}{25-T_{\infty}}$.
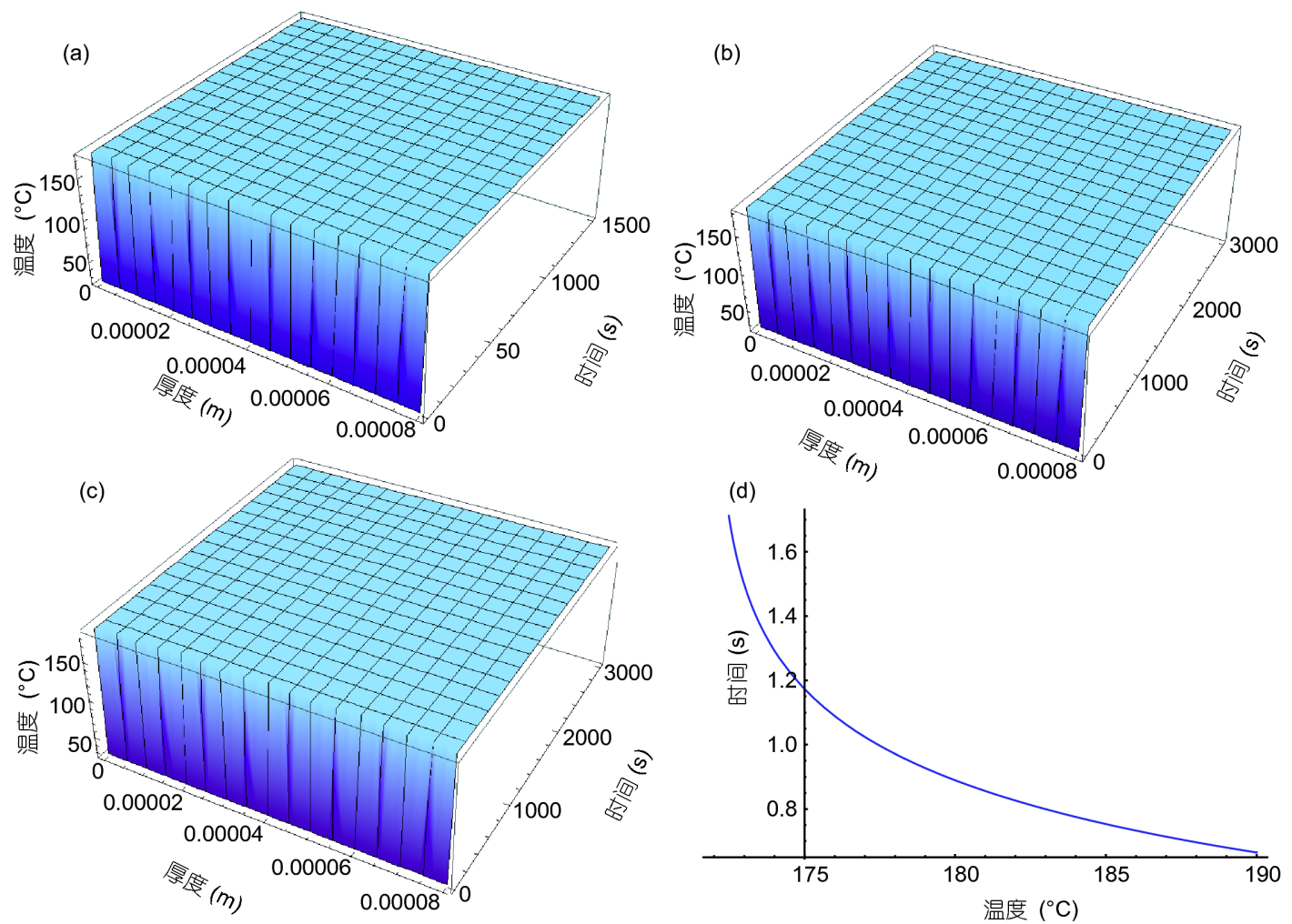

图 3 (网络版彩图)不同加热条件下阳极内部温度分布特征. (a) $T_{\infty}=175^{\circ} \mathrm{C}$; (b) $T_{\infty}=180^{\circ} \mathrm{C}$; (c) $T_{\infty}=185^{\circ} \mathrm{C}$; (d) 时间和温度的函数 关系 


\section{4 结果与讨论}

\section{1 实验结果及分析}

剥离率的计算是判断铜金属回收的关键指标. 利 用质量差来判断, 实验前测试阳极样品中阴极材料的 质量(利用样品总质量乘以阴极材料重量百分比获得, 其中阴极材料百分比通过初期的理化组成获得), 实验 后测试残留在样品上的阴极材料质量, 利用前后质量 变化率定义为剥离率. 基于正交实验设计和实验步骤, 对废锂离子电池中铜金属剥离回收, 得到不同条件下 的负极材料的剥离率, 结果如表4所示, 其各组实验剥 离后, 回收的铜金属如图4所示.

随着离子液体加热的进行, 温度逐渐接近PVDF黏 结剂的熔点, PVDF熔化导致铜簿与负极材料之间剥 离. 在外力搅拌作用下, 加快负极材料与铜䇴的分离 过程. 该过程主要利用热熔化的物理过程，目的是实 现铜金属高品位、纯单质态的回收，不引入新的杂质. 根据图4可直观观察到前3组有大量的碳粉仍黏滞在铜 䈃上未被除去，而4 6组稍好一些，7 9组已基本将碳 粉全部去除. 加热温度 $180^{\circ} \mathrm{C}$, 摚拌速度越大, 停留时 间越长, 越有利于二者之间的分离. 因而, 如果控制适 宜的加热温度、停留时间、搅拌速度, 可以获得高纯 度的铜金属, 验证技术工艺过程的可行性.

\section{2 数据分析}

对正交实验得到的结果采用方差分析法进行分 析, 可以得到如下数据(表5).

计算结果表明, 加热温度、停留时间、搅拌速度

表 4 实验结果

\begin{tabular}{ccccccc}
\hline 实验序号 & $\begin{array}{c}\text { 加热温 } \\
\text { 度 }\left({ }^{\circ} \mathrm{C}\right)\end{array}$ & $\begin{array}{c}\text { 停留时 } \\
\text { 间 }(\mathrm{min})\end{array}$ & $\begin{array}{c}\text { 摚拌 } \\
\text { 速度 } \\
(\mathrm{r} / \mathrm{min})\end{array}$ & $\begin{array}{c}\text { 摚拌前 } \\
\text { 质量 }(\mathrm{g})\end{array}$ & $\begin{array}{c}\text { 摚拌后量 }(\mathrm{g}) \\
\text { 剥离率 } \\
(\%)\end{array}$ \\
\hline 1 & 170 & 20 & 250 & 1.9662 & 0.9977 & 61.1 \\
2 & 170 & 25 & 300 & 1.9848 & 0.9023 & 67.6 \\
3 & 170 & 30 & 350 & 1.9458 & 0.7227 & 78.0 \\
4 & 175 & 20 & 300 & 1.9469 & 0.6712 & 81.2 \\
5 & 175 & 25 & 350 & 1.9641 & 0.6409 & 83.6 \\
6 & 175 & 30 & 250 & 1.9026 & 0.6228 & 83.4 \\
7 & 180 & 20 & 350 & 1.8954 & 0.5868 & 85.6 \\
8 & 180 & 25 & 250 & 1.938 & 0.5008 & 92.0 \\
9 & 180 & 30 & 300 & 1.965 & 0.519 & 91.3 \\
\hline
\end{tabular}

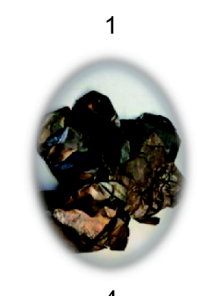

4

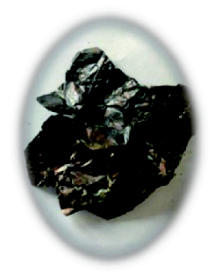

7

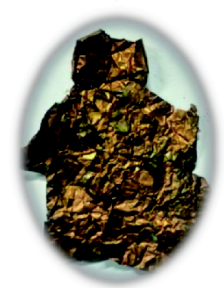

图 4 (网络版彩图)不同条件下各组的剥离外观图

表 5 正交实验方差分析

\begin{tabular}{|c|c|c|c|c|}
\hline 实验序号 & $\begin{array}{c}\text { 加热温度 } \\
\left({ }^{\circ} \mathrm{C}\right)\end{array}$ & $\begin{array}{c}\text { 停留时间 } \\
\text { (min) }\end{array}$ & $\begin{array}{c}\text { 搅拌速度 } \\
(\mathrm{r} / \mathrm{min})\end{array}$ & 剥离率(\%) \\
\hline 1 & 170 & 20 & 250 & 61.1 \\
\hline 2 & 170 & 25 & 300 & 67.6 \\
\hline 3 & 170 & 30 & 350 & 78.0 \\
\hline 4 & 175 & 20 & 300 & 81.2 \\
\hline 5 & 175 & 25 & 350 & 83.6 \\
\hline 6 & 175 & 30 & 250 & 83.4 \\
\hline 7 & 180 & 20 & 350 & 85.6 \\
\hline 8 & 180 & 25 & 250 & 92.0 \\
\hline 9 & 180 & 30 & 300 & 91.3 \\
\hline $\mathrm{K} 1$ & 1.667 & 1.838 & 1.908 & \\
\hline $\mathrm{K} 2$ & 2.002 & 1.961 & 1.936 & \\
\hline $\mathrm{K} 3$ & 2.168 & 2.038 & 1.993 & \\
\hline $\mathrm{k} 1$ & $68.9 \%$ & $76.0 \%$ & $78.9 \%$ & \\
\hline $\mathrm{k} 2$ & $82.7 \%$ & $81.1 \%$ & $80.0 \%$ & \\
\hline k3 & $89.6 \%$ & $84.2 \%$ & $82.4 \%$ & \\
\hline 极差 & $6.9 \%$ & $8.3 \%$ & $3.5 \%$ & \\
\hline $\begin{array}{c}\text { 因素影响主 } \\
\text { 次顺序 } \\
\end{array}$ & \multicolumn{3}{|c|}{ 停留时间 $>$ 加热温度 $>$ 搅拌速度 } & \\
\hline 优水平 & 180 & 30 & 350 & \\
\hline 优组合 & \multicolumn{3}{|c|}{$\left(180^{\circ} \mathrm{C}, 30 \mathrm{~min}, 350 \mathrm{r} / \mathrm{min}\right)$} & \\
\hline
\end{tabular}


可以不同程度地影响离子液体的剥离效果. 而其中, 停 留时间(极差为 $8.3 \%$ )和加热温度(极差为 $6.9 \%$ )两项影 响较大, 搅拌速度因素(极差为 $3.5 \%$ )相对影响不大. 且 在设定条件下，最优组合为: 加热温度 $180^{\circ} \mathrm{C}$ 、停留时 间为 $30 \mathrm{~min}$, 搅拌速度为 $350 \mathrm{r} / \mathrm{min}$. 为了对其进行验证, 在该条件下进行实验，计算负极材料的回收率达到 95\%, 铜金属可以得到全部回收，其纯度达到 $97 \%$ 以上 (纯度根据负极材料的残留或铜䈃的剥离率而定, 其它 杂质为残留的负极材料).

\section{3 分析讨论}

相比正极材料和铝金属回收来说，目前对废锂离 子电池中铜回收的研究不多. 本研究的铜金属回收率 可达 $100 \%$ ，纯度超过 $97 \%$ ，均高于 $\mathrm{da}$ Costa等人 ${ }^{[18]}$ 的 $54 \%$ 和 $66 \%$, Granata等人 ${ }^{[19]}$ 的 $78 \%$ 和 $95 \%$, Zhu等人 ${ }^{[20]}$ 的 $93.1 \%$ 和 $95.4 \%$ (图5). 主要原因在于, 过去的这些研 究通常采用破碎、分选的方式对废锂离子电池回收, 铜䈃在破碎时，不仅发生粒度减小、与负极材料脱离, 也会发生卷曲. 卷曲的铜箔将负极材料包裏在内, 很难 再进行分离，因而降低了铜金属的回收纯度和回收效 率. 另外, 在不同粒度的物料中, 均含有不同浓度铜金 属，过去这些研究只选择一定粒径物料进行资源化处

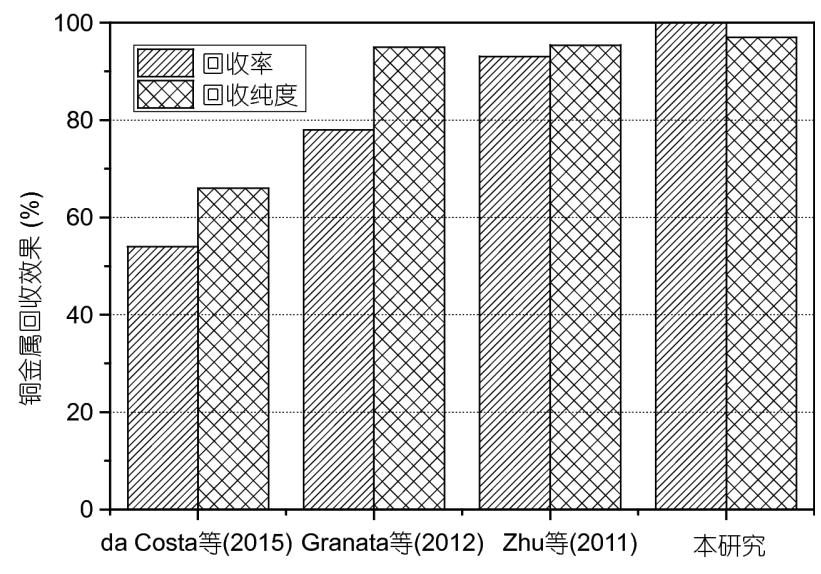

图 5 铜金属回收效果对比

理, 则降低了铜金属的回收率.

4.4 短程高效回收废理离子电池中有色金属的工 艺技术构建及分析

上述研究发现，离子液体加热可有效剥离废锂离 子电池阳极部分的铜䈃和负极材料. 基于实验结果和 已有研究，利用离子液体加热短程高效回收废锂离子 电池中有色金属的工艺技术如图6所示. 首先, 废锂离 子电池经过盐水放电和干燥，为了更高效回收有价材

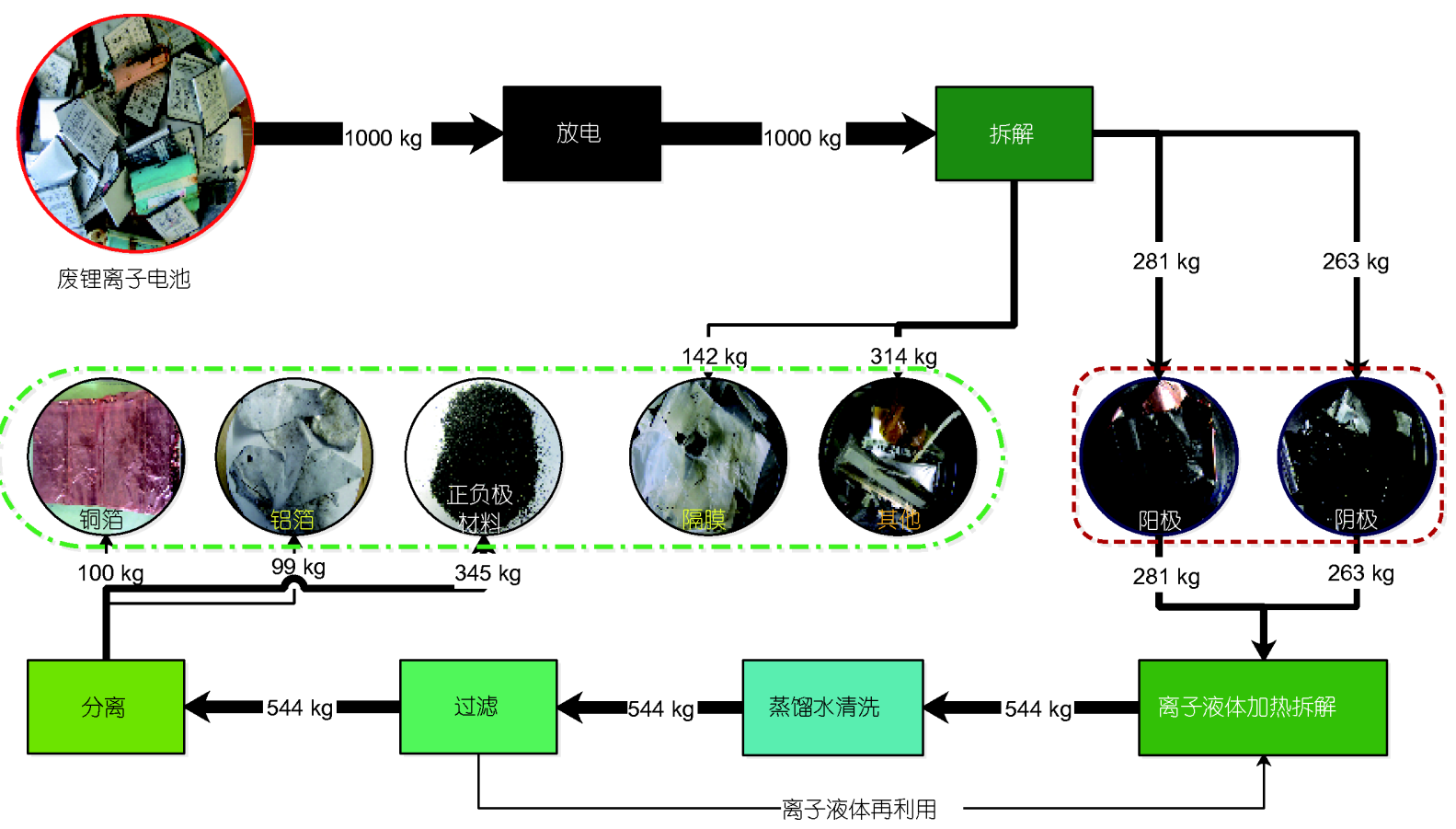

图 6 短程高效回收废锂离子电池中有色金属的工艺技术 
料, 对废电池进行手工拆解, 可以得到阴极、阳极、隔 膜等. 含有铜与碳粉的阳极和含有铝与钴酸锂材料的 阴极, 分别置于 $180^{\circ} \mathrm{C}$ 离子液体中, 调整摚拌机转速 $300 \sim 350 \mathrm{r} / \mathrm{min}$ ，保持停留时间25 30 min完成分离处 理. 最后, 离子液体进行冷却, 将高品位的铜簿、铝箔 和正极材料进行分离和清洗，最终获得高纯度的铜金 属、铝金属、正极材料和负极材料, 实现全组分回收. 含有离子液体的清洗液可进行重复使用, 因为 $[\mathrm{BMIm}]\left[\mathrm{BF}_{4}\right]$ 离子液体为水溶性的. 含有清洗水的离 子液体重新置入油浴锅后, 当温度加热至 $100^{\circ} \mathrm{C}$ 时, 首 先是水分蒸发, 完毕后继续升温至工艺最佳温度. 几乎 任何主流的正负极材料都可通过该工艺回收，不管正 极材料是钴酸锂还是其他物质。既然清洁的金属和正 负极材料可得到回收，这为这些材料的再利用、再循 环提供了可能.

从物质流分析角度看，根据构建的回收工艺和各 种废锂离子电池理化组成 ${ }^{[5]}, 1000 \mathrm{~kg}$ 混合废锂离子电
池经过放电、拆解、离子液体加热拆解、分离等过 程, 可以回收大约 $100 \mathrm{~kg}$ 铜箔、 $99 \mathrm{~kg}$ 铝䈃、 $345 \mathrm{~kg}$ 的 正负极材料和 $142 \mathrm{~kg}$ 隔膜等资源.

\section{5 结论}

(1) 离子液体加热处理废锂离子电池阳极过程中, 主要发生热对流和热传导过程; 利用摚拌机快速运转 可强化热对流的过程和机械力剥离极材料; 基于傅里 叶定律对该剥离过程建立了热传递过程数学模型.

（2）实验分析验证采用离子液体加热回收铜金属 的适宜工艺条件为: 加热温度 $180^{\circ} \mathrm{C}$ 、搅拌速度 $350 \mathrm{r} / \mathrm{min}$ 、停留时间 $30 \mathrm{~min}$, 经过处理后, 铜金属回收 率可达 $100 \%$, 纯度超过 $97 \%$.

(3) 根据研究结果, 提出了针对废锂离子电池的系 统化的短程高效回收工艺: 放电预处理、拆解、离子 液体加热分离、清洗、过滤/回用和最终分离等过程.

\section{参考文献}

1 Kim H, Jang Y C, Hwang Y, et al. End-of-life batteries management and material flow analysis in South Korea. Front Environ Sci Eng, 2018, 12: 3

2 Pehlken A, Albach S, Vogt T. Is there a resource constraint related to lithium ion batteries in cars? Int J Life Cycle Assess, 2017, 22: 40-53

3 Zeng X, Li J, Ren Y. Prediction of various discarded lithium batteries in China. In: 2012 IEEE International Symposium on Sustainable Systems and Technology (ISSST). Boston, 2012

4 Gies E. Recycling: Lazarus batteries. Nature, 2015, 526: S100-S101

5 Zeng X, Li J. Spent rechargeable lithium batteries in e-waste: Composition and its implications. Front Environ Sci Eng, 2014, 8: 792-796

6 Wang M, Tan Q, Chiang J F, et al. Recovery of rare and precious metals from urban mines-A review. Front Environ Sci Eng, 2017, 11: 1-17

7 Chen J, Li Q, Song J, et al. Environmentally friendly recycling and effective repairing of cathode powders from spent $\mathrm{LiFePO}_{4}$ batteries. Green Chem, 2016, 18: 2500-2506

8 Wang M M, Zhang C C, Zhang F S. An environmental benign process for cobalt and lithium recovery from spent lithium-ion batteries by mechanochemical approach. Waste Manage, 2016, 51: 239-244

9 Zhang X, Xue Q, Li L, et al. Sustainable recycling and regeneration of cathode scraps from industrial production of lithium-ion batteries. ACS Sust Chem Eng, 2016, 4: 7041-7049

10 Gao W, Zhang X, Zheng X, et al. Lithium carbonate recovery from cathode scrap of spent lithium-ion battery: A closed-loop process. Environ Sci Technol, 2017, 51: 1662-1669

11 Xiao J, Li J, Xu Z. Novel approach for in situ recovery of lithium carbonate from spent lithium ion batteries using vacuum metallurgy. Environ Sci Technol, 2017, 51: 11960-11966

12 Zeng X, Li J, Singh N. Recycling of spent lithium-ion battery: A critical review. Critical Rev Environ Sci Tech, 2014, 44: 1129-1165

13 Zhang X, Xie Y, Lin X, et al. An overview on the processes and technologies for recycling cathodic active materials from spent lithium-ion batteries. J Mater Cycle Waste Manag, 2013, 15: 420-430

14 张锁江, 刘晓敏, 姚晓倩, 等. 离子液体的前沿、进展及应用. 中国科学B辑: 化学, 2009, 39: 1134-1144

15 Zeng X, Li J. Innovative application of ionic liquid to separate Al and cathode materials from spent high-power lithium-ion batteries. J Hazard 
Mater, 2014, 271: 50-56

16 Holomb R, Martinelli A, Albinsson I, et al. Ionic liquid structure: The conformational isomerism in 1-butyl-3-methyl-imidazolium tetrafluoroborate $\left([\mathrm{bmim}]\left[\mathrm{BF}_{4}\right]\right)$. J Raman Spectrosc, 2008, 39: 793-805

17 东莞国佳. PVDF塑胶原料(poly vinylidene fluoride)-聚偏氟乙烯. 2014 [2014-02-23]. http://www.gjsjyl.com/hydt/2013/0822/418.html

18 da Costa A J, Matos J F, Bernardes A M, et al. Beneficiation of cobalt, copper and aluminum from wasted lithium-ion batteries by mechanical processing. Int J Mineral Process, 2015, 145: 77-82

19 Granata G, Moscardini E, Pagnanelli F, et al. Product recovery from Li-ion battery wastes coming from an industrial pre-treatment plant: Lab scale tests and process simulations. J Power Sources, 2012, 206: 393-401

20 Zhu S G, He W Z, Li G M, et al. Recovering copper from spent lithium ion battery by a mechanical separation process. In: 2011 International Conference on Materials for Renewable Energy \& Environment (ICMREE). Shanghai, 2011. 1008-1012

\title{
Short-cut and high-efficiency process to recycle copper from spent lithium-ion battery
}

\author{
JULDEZ Nurlan, LI JinHui \& ZENG XianLai
}

Key Laboratory for Solid Waste Management and Environment Safety, School of Environment, Tsinghua University, Beijing 100084, China

The booming flourish of information communication technology and electric vehicle industry has resulted in plenty of spent lithiumion battery $(\mathrm{LiB})$. Its recycling is recognized as the vital approach for resource sustainability and environmental improvement. However, the conventional recycling process was much complicated with a low recycling efficiency. We chose ionic liquid as heat medium to melt the binder and separate copper foil and anode material. Theoretical analysis based on Fourier's law was adopted to determine the heat transfer mechanism of cathode material and to examine the relationship between heating temperature and retention time. All the experimental and theoretic results show that peel-off rate of copper foil from anode materials could reach $95 \%$ when major process parameters were controlled at $180^{\circ} \mathrm{C}$ heating temperature, $350 \mathrm{r} / \mathrm{min}$ agitator rotation, and $30 \mathrm{~min}$ retention time. The obtained results further imply that the application of ionic liquid for recycling copper and anode materials from spent LiBs is short cut and highly efficient, regardless of the application source of the LiBs or the types of anode material. The proposed process will smooth the high recycling rate for copper and aluminum, and enable the closed-loop supply chain for spent lithium-ion battery.

spent lithium-ion battery, recycling, copper, ionic liquid, Fourier's law

doi: $10.1360 /$ N092017-00385 